\title{
On the Urban Comprehensive Carrying Capacity of the Ethnic Regions in China
}

\author{
Peng $\mathrm{Li}^{1} \&$ Haiying $\mathrm{Ma}^{1}$ \\ ${ }^{1}$ School of Economics, Northwest University for Nationalities, Lanzhou, China \\ Correspondence: Haiying Ma, School of Economics, Northwest University for Nationalities, Lanzhou 730124, China. \\ E-mail: lxmahaiying8888@163.com
}

Received: March 28, 2014

Accepted: April 9, 2014

Online Published: April 14, 2014

doi:10.5430/ijfr.v5n2p189

URL: http://dx.doi.org/10.5430/ijfr.v5n2p189

This work was supported by the Fundamental Research Funds for the Central Universities of Northwest University for Nationalities (Grant No: ZYZ2011013).

\begin{abstract}
In this paper, we analyzed a fresh conception of the urban comprehensive carrying capacity in the context of ecosystem, economy and social dimensions. Based on the AHP methodology, the author constructs the urban comprehensive carrying capacity hierarchical structure model for the chosen ten regions in Inner Mongolia, China. The findings are a better reflection that the urban comprehensive carrying capacity is a complex chaos interconnected with the ecological, economic and social throughputs of the development-oriented regions, and it is an entangled combination of three self-organizing non-equilibrium systems with all concepts fundamental to sustainable urbanization process.
\end{abstract}

Keywords: AHP, ethnic regions, comprehensive carrying capacity, analytical hierarchy process

\section{Introduction}

Urbanization is a typical epoch evolved along with industrial and technological revolutions, and a process of clusters of production factors and culture charms, consequently leads to the explicit and ineradicable changes in society associated with economic structure, space layout of rural and urban areas, living styles and behaviors of the new inhabitants, and the development of large-scale coordinated activities etc. China has adopted urbanization strategy since the year 2000 and has achieved enormous attainments, with 0.622 billion urban population by the end of 2009 . The urbanization rate ascends to $46.6 \%$, averaged at $0.9 \%$ since 2006 --the starting point of the Outline of China's the Eleventh Five-year Plan for Economic and Social Development. The ethnic regions are the core part of urbanization strategy of China and may exert direct effects on the overall urbanization system of China. Endowed with rich natural resources and vulnerable ecosystem, accompanied with cultural complexity, poverty and high rate if illiteracy, the ethnic regions owe a comparatively slower pace of urbanization.

This study is focused on controversies surrounding the process of urbanization that more and more scholars have recognized the limitations and constraints of the carrying capacity of the cities, yet they are rather confined within the one-sidedness of the research from the single-element perspective in ecosystem, environment, resources as land, water or talents etc. The main contributions of this paper are: (1) The conception of the urban comprehensive carrying capacity is redefined as an entanglement of ecological carrying capacity, economic carrying capacity and social carrying capacity and discussed in depth. (2) The urban comprehensive carrying capacity hierarchical structure model is constructed, which outlines the key indicators in three differentiated layers and pioneers in the research perceptions. The first section of the study encompasses an introduction to the conceptual framework of the comprehensive carrying capacity, and lists its enriched definition with ecological, economic and social ties.

\section{The Framework of the Urban Comprehensive Carrying Capacity}

\subsection{Biological Carrying Capacity}

Briefly, the biological carrying capacity refers to the maximal number of individuals who can be supported in a given urban area within natural resource limits, or in a deeper sense, the maximal accommodation ability of the given region in the social and economic activities for such the maximal load. The biological carrying capacity are only conditional on future human choices and natural events, it may fluctuate along with the technological advancement, 
the mass migration etc.

\subsection{Economic Carrying Capacity}

The pace of the economy development of a specific urban region must be considered for an urban comprehensive carrying capacity. The urban economic carrying capacity refers to the notion that at what level of capabilities the resources within the chosen urban area can be effectively contributable to the economic development.

\subsection{Social Carrying Capacity}

A related rationale for carrying capacity is the social carrying capacity, which is a well-founded premise that despite our increasing technological sophistication, humankind remains in a state of obligate dependence on the productivity and life support services of the ecosphere. Thus, from an ecological perspective, adequate land and associated productive factors are fundamental for continued civilized existence on the Earth. However, the revival of many other elements should be cited in sustainable development planning, such as infrastructure, education, medical care, social security system etc.

\section{The Measurement Model}

\subsection{Theoretical Methods and Data}

The core philosophy of AHP consists of three stages. Firstly, define clearly the final objective of the research issue, and then regroup the concerned sub-issues. Secondly, make a general assessment to the importance of different hierarchy clusters subject to the appraisals from the research scholars and the results of the statistical calculation, thus conclude the weight value for respective hierarchy clusters. Last, recognize the perceptual analysis and make necessary adjustments to internalize the data available for effective use. Based on the data from statistical yearbook 2009 of Inner Mongolia and other relative figures from the websites of local governments, the author redefines the determinants of the urban comprehensive carrying capacity in three hierarchy clusters as the final objective, directive criteria and the decisive indexes. The hierarchy of decisive indexes is a family of seventeen area-based sustainability indicators. Five indexes are closely interconnected with the ecological carrying capacity and listed as $\mathrm{C} 1$ the available water supply, C2 the available grassland, C3 industrial waste treatment rate, C4 percentage of forest cover, C5 number of environmental agencies. The referent group of factors for economic carrying capacity are C6 GDP per capita, C7 the proportion of tertiary industry output in GDP, C8 the average disposable income of the urban inhabitants, C9 Engel's coefficient of urban inhabitants, C10 the total investment in fixed assets, C11 employment percentage per household. The rest six indexes are measures of social carrying capacity with $\mathrm{C} 12$ urban built-up area, C13 total employment percentage of secondary\& tertiary industry, C14 number of ordinary middle school, C15 number of public library, $\mathrm{C} 16$ number of health agencies, $\mathrm{C} 17$ natural increase rate of population.

\subsection{The Measurement of Urban Comprehensive Carrying Capacity}

3.2.1 Indicators Non-dimensionalization. Since the seventeen indexes get different dimensions for fundamental measurement of the chosen research platforms such as percentage, land area, numerical figures and even monetary units, it is rather important to make indexes being dimensionless to facilitate calculations and deductions to achieve high accuracy and reliability. To make the following analysis perceptible enough, the possible resultant comprehensive carrying capacity will be ranging between $0-1$ on the redirected methodology, that is, the minimal indexes as $\min x_{i j}, x^{\prime}=1$, the maximal indexes as $\max x_{i j}, x^{\prime}=1$. The medians are classified as positive indicators and negative indicators, and the former are quantified as higher semi-trapezium fuzzy membership function as:

$$
x_{i j}^{\prime}=\frac{X_{i j}-\min X_{i j}}{\max X_{i j}-\min X_{I J}}
$$

While the latter as lower semi-trapezium fuzzy membership function as:

$$
X_{i j}^{\prime}=\frac{\max X_{i j}-X_{i j}}{\max X_{i j}-\min X_{i j}}
$$

where $X_{i j}$ stands for the index value of $\mathrm{j}$ factors while $X_{i j}^{\prime}$ as $\mathrm{j}$ factor scores ranging between $0-1$.

3.2.2 Construction of Judgment Matrix. An effective hierarchy structure can reflect the correlations of involving directive criteria, yet this intuitive hypothesis can never hint the equally considerable importance of different layers 
to the final objective, and obviously from the perspectives of policymakers, each element imposing differentiated forces to the ultimate result. While attempting to finalize the exact influencing forces of the listed factors supposed as $X=\left\{x_{1}, x_{2}, \cdots x_{n}\right\}$ to the target factor $\mathrm{Z}$ and obtain reliable and practical answers, we adopt the methodology proposed by Saaty and construct a pair-comparison matrix. Every time we pick out two factors $X_{i}$ and $X_{j}$, nominate $a_{i j}$ to represent the proportion between the pair of $X_{i}$ and $\mathrm{Z}$ and $X_{j}$ and $\mathrm{Z}$ in light of the influencing forces as in respective comparison cases. We then compute the total results by summing all the figures in a pair-comparison matrix $\mathrm{A}$ of all the relative $\mathrm{X}$ and $\mathrm{Z}$ in the following equation $A=\left(a_{i j}\right)_{n \times n}$, and the scales of $a_{i j}$ may be shown in the 1-9 level scale and their respective reciprocals.

On the supposition that $\lambda$ is the special radical, $\mathrm{W}$ is eigenvector, we may get the judgment matrix $\mathrm{HW}=\lambda \mathrm{W}$ and go further for the results of $\max (\lambda)$ and the corresponding $\mathrm{W}$, thus the weight values of directive criterion for the target after the normalization processing of the varied eigenvectors. We adopt consistency adjustment in the AHP model to avoid inconsistency of the judgment matrix $\mathrm{H}$, therefore the concept of consistency index is put into use.

While $\lambda_{\max }=n, \mathrm{CI}=0$, this implies the judgment matrix is perfectly consistent. The larger the figures of CI result, the lower consistency of the judgment matrix.

Besides the possibly subjective assessments of the participants, the proportion scaling of the pair comparison may affect the consistency of the judgment matrix. The varied acceptable values of CI for differentiated-order matrix demand the RI methodology in our research. That is, we apply the average random consistency index to make the consistency indexes revised so as to avoid the results deviating from the fact. The revised result CR should be

$$
C R=\frac{C I}{R I} \times 100 \%
$$

Generally, for judgment matrix of $n \geq 3$ order, it owes highly acceptable consistency in the case $C R \leq 0.1$. Whist $C R>0.1$ alleges the clear deviation from the harmonious consistency and further indicators amendment is thus indispensable for the satisfactory research results.

3.2.3 Resultant Calculation of Indicators. The concerned data from the assessments of the participant scholars and the answers of the questionnaires serve to illustrate application of the AHP concept. The pair-comparison judgment matrixes of the directive criteria in the AHP model are as follows.

The results of Table 1 are $\lambda \max =3, \mathrm{CI}=0, \mathrm{RI}=0.58$, and the relevant $\mathrm{CR}=0.079<0.1$, thus high acceptability in the consistency of the judgment matrix.

Table 1. A-B judgment matrix

\begin{tabular}{lcccc}
\hline A & B1 & B2 & B3 & Wi \\
\hline B1 & 1 & $1 / 3$ & 1 & 0.2 \\
\hline B2 & & 1 & 3 & 0.6 \\
\hline B3 & & & 1 & 0.2 \\
\hline
\end{tabular}

The results of Table 2 are $\lambda$ max $=5.352, \mathrm{CI}=0.088, \mathrm{RI}=1.120$, and the relevant $\mathrm{CR}=0.079<0.1$, thus highly acceptable consistency of the judgment matrix.

Table 2. B1-C judgment matrix

\begin{tabular}{lllllll}
\hline B1 & C1 & C2 & C3 & C4 & C5 & Wi \\
\hline C1 & 1 & 5 & 7 & 3 & 7 & 0.516 \\
\hline C2 & & 1 & 5 & 1 & 7 & 0.204 \\
\hline C3 & & 1 & $1 / 3$ & 3 & 0.068 \\
\hline C4 & & & 1 & 3 & 0.172 \\
\hline C5 & & & & 1 & 0.041 \\
\hline
\end{tabular}


The results of Table 3 are $\lambda \max =6.599, \mathrm{CI}=0.120, \mathrm{RI}=1.240$ and the relevant $\mathrm{CR}=0.097<0.1$, thus the judgment matrix still owes high acceptability in the consistency.

Table 3. B2-C judgment matrix

\begin{tabular}{lccccccc}
\hline B2 & C6 & C7 & C8 & C9 & C10 & C11 & Wi \\
\hline C6 & 1 & 2 & $1 / 3$ & 2 & $1 / 3$ & $1 / 7$ & 0.079 \\
\hline C7 & & 1 & 1 & $1 / 3$ & $1 / 5$ & $1 / 3$ & 0.059 \\
\hline C8 & & & 1 & 1 & $1 / 5$ & $1 / 5$ & 0.088 \\
\hline C9 & & & & 1 & $1 / 5$ & $1 / 5$ & 0.079 \\
\hline C10 & & & & & 1 & 1 & 0.337 \\
\hline C11 & & & & & & 1 & 0.357 \\
\hline
\end{tabular}

The results of Table 4 are $\lambda \max =6.562, \mathrm{CI}=0.112 \mathrm{RI}=1.240$ and the relevant $\mathrm{CR}=0.0903<0.1$, thus the judgment matrix possesses high acceptability in the consistency.

Table 4. B3-C judgment matrix

\begin{tabular}{lrrrrrrl}
\hline $\mathrm{B} 3$ & $\mathrm{C} 12$ & $\mathrm{C} 13$ & $\mathrm{C} 14$ & $\mathrm{C} 15$ & $\mathrm{C} 16$ & $\mathrm{C} 17$ & Wi \\
\hline $\mathrm{C} 12$ & 1 & 3 & 5 & 5 & 7 & 3 & 0.404 \\
\hline $\mathrm{C} 13$ & & 1 & 4 & 7 & 5 & 3 & 0.270 \\
\hline $\mathrm{C} 14$ & & & 1 & 5 & 4 & 3 & 0.142 \\
\hline $\mathrm{C} 15$ & & & & 1 & 2 & $1 / 3$ & 0.047 \\
\hline $\mathrm{C} 16$ & & & & & 1 & $1 / 3$ & 0.039 \\
\hline $\mathrm{C} 17$ & & & & & 1 & 0.099 \\
\hline
\end{tabular}

As indicated in the results of Tables 1 to 4, all of the single values of CR are below 0.1 , therefore the weight distribution in the judgment matrixes has the best consistency. We seek for further test of the consistency indices for the levels of total order based on the following equation.

$$
C R_{\text {sum }}=\frac{\sum_{j=1}^{n} a_{j} C I_{j}}{\sum_{j=1}^{n} a_{j} R I_{j}}
$$

On conditions that $\mathrm{CI}=0.112, \mathrm{RI}=1.216, \mathrm{CRsum}=0.092<0.1$, the Table 5 has acquired satisfactory feasibility and validity.

Table 5. Weight distribution of index

Layer $\mathrm{C}$ to target Layer A

\begin{tabular}{rllll}
\hline \multirow{2}{*}{$\mathrm{A}$} & $\mathrm{B} 1$ & $\mathrm{~B} 2$ & $\mathrm{~B} 3$ & $\mathrm{Wi}$ \\
\cline { 2 - 5 } & 0.2 & 0.6 & 0.2 & 0.103 \\
\hline $\mathrm{C} 1$ & 0.516 & & & 0.041 \\
\hline $\mathrm{C} 2$ & 0.204 & & & 0.014 \\
\hline $\mathrm{C} 3$ & 0.068 & & & 0.034 \\
\hline $\mathrm{C} 4$ & 0.172 & & 0.008 \\
\hline $\mathrm{C} 5$ & 0.041 & & & 0.048 \\
\hline $\mathrm{C} 6$ & & 0.079 & & 0.036 \\
\hline $\mathrm{C} 7$ & 0.059 & & 0.053 \\
\hline $\mathrm{C} 8$ & 0.088 & & 0.047 \\
\hline $\mathrm{C} 9$ & 0.079 & & 0.202 \\
\hline $\mathrm{C} 10$ & 0.337 & 0.404 & 0.214 \\
\hline $\mathrm{C} 11$ & 0.357 & 0.270 & 0.081 \\
\hline $\mathrm{C} 12$ & & 0.142 & 0.054 \\
\hline $\mathrm{C} 13$ & & & 0.047 & 0.028 \\
\hline $\mathrm{C} 14$ & & 0.039 & 0.009 \\
\hline $\mathrm{C} 15$ & & 0.099 & 0.008 \\
\hline $\mathrm{C} 16$ & & & 0.020 \\
\hline $\mathrm{C} 17$ & & & \\
\hline
\end{tabular}


Table 6. Evaluation sort of the urban comprehensive carrying capacity

\begin{tabular}{lllllllllll}
\hline Regions & $\begin{array}{l}\text { Hulu } \\
\mathrm{n} \\
\text { BuirC }\end{array}$ & $\begin{array}{l}\text { Xing'n } \\
\text { League }\end{array}$ & $\begin{array}{l}\text { Tonglia } \\
\mathrm{o}\end{array}$ & Chifeg & $\begin{array}{l}\text { XilinG } \\
\text { olLeag } \\
\text { ue }\end{array}$ & Wulanchau & Erdos & Wuhi & $\begin{array}{l}\text { Alxa } \\
\text { Leage }\end{array}$ & $\begin{array}{l}\text { Baya } \\
\text { nnr }\end{array}$ \\
\hline $\begin{array}{l}\text { Total } \\
\text { Value }\end{array}$ & 0.574 & 0.2552 & 0.3805 & 0.3693 & 0.2634 & 0.1890 & 0.749 & 0.281 & 0.213 & 0.326 \\
\hline $\begin{array}{l}\text { Evaluati } \\
\text { n Sort }\end{array}$ & 2 & 8 & 3 & 4 & 7 & 10 & 1 & 6 & 9 & 5 \\
\hline
\end{tabular}

On the solid foundations of the previous calculations and deductions for the weight distributions of each judgment matrix, the total value, and the further evaluation sort of the urban comprehensive carrying capacity of the ten chosen regions of Mongolia is defined, using the weight sum method for the set of normalized data.

3.2.4 Analyses Reflections. The present and related analysis confirms that Erdos city ranks first in its urban comprehensive carrying capacity. This owes greatly to the reason that the economic carrying capacity of Erdos city is rather strong, yet the ecological carrying capacity and social carrying capacity may, to some extent, hinder its urbanization process. Hulun Buir city, Tongliao city and Chifeng city rank the second, the third and the forth position in their urban comprehensive carrying capacity respectively. The majorities of the directive indices are at the medium range, while a few are below the average and encumber the resultant level, thus may be obstacles of the urbanization. Bayannur city and Wuhai city are the fifth and sixth in the urban comprehensive carrying capacity. With few indices lingering at the medium range and great majority nearly hitting the rock-bottom, Xilin Gol league, Xing'an league, Alxa league and Wulanchabu city rank the seventh, eighth, ninth and tenth in our list.

\section{Conclusions}

The Analytical Hierarchy Process provides a useful tool for the concept of urban comprehensive carrying capacity, as it is an entangled chaos of three independent ecological, economic and social subsystems, which may all induce the negative feedbacks and consequences accrued from population shift along with the irrevocable urbanization. This fresh-defined analysis supports argument to shift the emphasis in urbanization process from production factors integration, coordination of economy structure and space layout, to inter-regional dependency and intra-regional self-reliance, as the aggregate effect would be stability in ecosystem, economic leap and social welfare.

\section{References}

Chen, Yuzhen, \& Nie, Hua. (2010). Dynamics of ecological footprint and ecological capacity of Fujian province. Science \& Technology Information, (9), 171-172. (in Chinese)

Dhondt A A. (1988). Carrying capacity: a confusing concept. Acta Oecologica/ Oceologia Generalis, 9(4), 337-346.

Liu, Xiaorong, Cao, Fang, \& Du, Ying. (2010). Ecological capacity of Minqin oasis in fragile region based on ecological footprints theory. Journal of Arid Land Resources and Environment, 32-36. (in Chinese)

Mao, Hanying, \& Yu, Danlin. (2001, Aug.). A study on the quantitative research of regional carrying capacity. Advance in Earth Sciences, 16(4), 549-555. (in Chinese)

Meyer, P.S., \& Ausubel, J.H. (1999). Carrying capacity: a model with logically varying limits. Technological Forecasting and Social Change, 61(3), 209-214.

Odum, E.P. (1953). Fundamentals of Ecology. Philadelphia: W. B. Saunders.

Zhou, Chun, Shu, Tingfei, \& Wu, Renhai. (2003). A study of carrying capacity of land resources in Pearl River Delta. Management Geological Science and Technology, (6), 16-19. (in Chinese) 